\title{
Costing analysis of field implementation of hepatitis $C$ case detection in rural Maung Russey operational district, Cambodia
}

Su Myat Han,, ,b Ir Por, ${ }^{c}$ Keo Samley, ${ }^{d}$ Voeurng Bunreth, ${ }^{e}$ Chris Smith, ${ }^{b}$ Koya Ariyoshi, ${ }^{b, f}$ Jean-Philippe Dousseta and Mickael Le Paiha

Correspondence to Su Myat Han (email: pearl.june@gmail.com)

Background: When a new health programme is introduced, it is crucial to estimate the costs for rational health policy decision-making. The aim of this study was to determine the costs of implementing two strategies for hepatitis $\mathrm{C}$ virus (HCV) screening in rural Cambodia.

Methods: We retrospectively analysed clinical and cost data that were collected routinely for a demonstration project for scaling up HCV screening and testing in Cambodia. The programme data were collected between March and December 2018 in Maung Russey operational district in Battambang Province, Cambodia.

Findings: During the study period, 24230 people were screened; 1194 (5\%) were HCV seropositive, of whom 793 $(66 \%)$ were confirmed to be viraemic. During the study period, $18 \%$ of the estimated population of the operational district were screened, of whom $45 \%$ were estimated to be seropositive and $41 \%$ to be viraemic. With passive screening alone, $8 \%$ of the estimated population were screened, of whom $29 \%$ were estimated to be seropositive and $28 \%$ viraemic. The cost per detected viraemic case was US\$ 194 for passive screening alone and US\$ 283 for passive and active screening combined. Labour costs (31\%) and tests and materials (29\%) comprised the largest proportions of the cost.

Conclusion: Combined active and passive screening per viraemic case detected was US $\$ 89$ more expensive than passive screening alone but provided a higher yield (41\% versus $28 \%$ ) of viraemic cases. Therefore, adding active screening to passive screening is beneficial. Selective active screening strategies, such as targeting people over 45 years and other higher-risk groups, added value for HCV diagnosis.

A n estimated 71 million people globally are living with chronic hepatitis $\mathrm{C}$ virus (HCV) infection, but $<20 \%$ of these individuals are aware of their infection status. ${ }^{1}$ Although affordable directly acting antivirals are available to treat $\mathrm{HCV}^{2}$ finding cases remains difficult, obviating increasing access to $\mathrm{HCV}$ treatment, especially in low- and middle-income countries such as Cambodia.

It has been estimated that about $2 \%$ of the population of Cambodia is living with chronic HCV infection, with a higher prevalence in older age groups. ${ }^{3-6}$ Genotypes 1 and 6 were the main HCV genotypes in the country, each at about $46 \%$ prevalence. ${ }^{7}$ Liver cancer is the leading cause of cancer deaths in the country, with $76 \%$ attributed to hepatitis B and/or HCV infection. ${ }^{8,9}$ In a seroprevalence survey conducted in Maung Russey health district in Cambodia, about $65 \%$ of the population was unaware of $\mathrm{HCV}^{6}$

In early 2020, the Cambodia Ministry of Health established a national strategic plan (2020-2024) and national guidelines for viral hepatitis $\mathrm{B}$ and $\mathrm{C},{ }^{10}$ which includes scaling up HCV testing nationwide; however, plans for screening have not yet been issued. Recommendations on the cost and effectiveness of

Médecins Sans Frontières - France, Phnom Penh, Cambodia.

Department of Tropical Medicine and Global Health, Nagasaki University, Japan.

National Institute of Public Health, Cambodia.

Communicable Disease Control Department, Ministry of Health, Cambodia.

Provincial Health Department, Battambang, Ministry of Health, Cambodia.

Department of Clinical Medicine, Nagasaki University, Japan.

Published: 12 July 2021

doi:10.5365/wpsar.2020.11.3.006 
different screening strategies for HCV are therefore necessary to ensure effective HCV service delivery and management in Cambodia and in countries with similar settings. Population or mass screening has been used in countries such as Egypt and Rwanda; ${ }^{11,12}$ however, high costs are a potential drawback to universal programmes.

In Cambodia, the medical humanitarian organization Médecins Sans Frontières (MSF) has been providing free HCV screening and treatment in collaboration with the Ministry of Health in Phnom Penh since September 2016 and in Battambang Province since March 2018. The projects were managed in partnership with the Department of Communicable Disease Control. By the end of 2020, 130327 people had been screened in Cambodia, of whom 30684 (22\%) were found to be HCV seropositive; of those, 20645 (70\%) were HCV viraemic, and 18900 had initiated treatment with directly acting antivirals.

The aim of the current study was to evaluate the cost and effectiveness of two screening strategies in order to inform public health authorities for future national screening.

\section{METHODS}

\section{Study setting}

Data routinely collected for an HCV programme in Maung Russey, Cambodia, between March and December 2018 were analysed. Maung Russey is a rural operational district with an estimated population of 209949 distributed in 175 villages in 2018. An MSF project for HCV screening and treatment was launched with existing Ministry of Health services, which included 13 health centres and a referral hospital covering the entire Maung Russey district.

\section{Screening strategies}

Two screening strategies were used to identify HCV cases in the community: passive and active screening. Passive screening covered the general population aged $\geq 18$ years and comprised voluntary HCV testing at health centres. This was initiated in March 2018 and continued for the duration of the study period.
In September 2018, after six months of passive screening and to increase case detection, active screening was added. Active screening targeted people aged $\geq 45$ years, as epidemiological studies indicated that this age group is at higher risk of HCV infection than others. ${ }^{4,6}$

The implementation team comprised an MSF nurse, health centre staff, village chiefs and community volunteers. Information on screening activities and awareness about HCV were provided to the community before HCV screening days by village and community leaders through a village information-sharing system for one half day. On the screening day, all seropositive cases were referred to their closest health centre for confirmatory testing of viral load. The active screening strategy comprised one day of screening per village between September and December 2018 in the 175 villages and sub-villages in the Maung Russey operational district. Passive screening continued during this time.

In both strategies, screening of people who were under tuberculosis (TB) treatment was postponed until the end of their treatment. People known to be living with HIV were referred to the National Centre for HIV/ AIDS, Dermatology and Sexually Transmitted Diseases. Screening of pregnant or breastfeeding women was deferred until the end of breastfeeding. There was no overlap of the passive and active programmes, as all people were systematically asked by the screening team whether they had already been screened and/or treated for HCV infection.

\section{Screening test and diagnosis}

The rapid diagnostic test SD Bioline ${ }^{\circledR}$ was used for diagnosis in both screening strategies on capillary blood collected by fingerprick by trained nurses. Positive cases were considered HCV seropositive. Confirmatory testing was performed with a viral load test based on a polymerase chain reaction assay for HCV RNA. Cases positive in this test were considered viraemic positive cases.

From seropositive patients detected in the passive screening programme, venous blood was collected for a viral load test at a health centre. Seropositive patients detected by active screening were referred to the nearest 
health centre for blood collection. The $2 \%$ of referred patients who did not show up for the viral load test were actively followed up by a phone call from an MSF nurse.

The collected blood samples were transported to the referral hospital for confirmatory assays. Collected specimens were centrifuged on the same day and transported via cold chain $\left(2-8{ }^{\circ} \mathrm{C}\right)$ to the Maung Russey hospital laboratory. Samples not processed for analysis within 24 hours were stored in a refrigerator $\left(2-8{ }^{\circ} \mathrm{C}\right)$. The GeneXpert ${ }^{\circledR}$ HCV viral load assay was used.

\section{Screening effectiveness}

The "number needed to screen" (NNS) for diagnosis of one viraemic case was calculated as the number of screened people divided by the number of confirmed HCV viraemic cases.

To compare the effectiveness of the two screening strategies (passive versus passive plus active screening), three outcomes were assessed: (i) the number of serological tests performed as a percentage of the target population; (ii) the number of positive serological cases as a percentage of the estimated number of $\mathrm{HCV}$ cases derived from a previous seroprevalence survey; ${ }^{4}$ and (iii) the number of viraemic cases as a percentage of the estimated number of HCV cases. The denominators of each outcome were as follows.

i. The targeted population was estimated from the 2013 Cambodia intercensal population survey. ${ }^{13}$ The estimated targeted populations were 133162 people aged $\geq 18$ years and 41823 aged $\geq 45$ years.

ii. The numbers of seropositive cases were estimated to be 2663 ( $\geq 18$ years) and 2133 ( $\geq 45$ years) in accordance with the results of a previous seroprevalence survey, for seropositivity rates of $2.6 \%$ ( $\geq 18$ years) and $5.1 \%$ ( $\geq 45$ years).

iii. The numbers of viraemic cases were estimated to be 1943 ( $\geq 18$ years) and 1514 ( $\geq 45$ years) in accordance with the previous seroprevalence survey of a prevalence of viraemia of $1.9 \%$ in the general population and 3.6\% among people $\geq 45$ years.

\section{Costing analysis}

Data were collected on expenditures for both passive and active screening, and data on the salaries of Ministry of Health and MSF staff involved in HCV screening were collected as a component of labour costs. Expenditure for screening included training and capacity-building, equipment (including depreciation), labour (for project personnel and health facility staff as opportunity costs), travel, materials and tests, user fees (MSF paid for uninsured patients to access other tests at the same time as HCV testing) and indirect costs considered to be associated with HCV screening, including overhead costs of the MSF office in Maung Russey, the Maung Russey referral hospital and health centres. The mean cost per HCV case identified was calculated by summing the cost of all resources used for screening and dividing them by the number of viraemic cases diagnosed. Costs are expressed in US dollars.

\section{RESULTS}

\section{Screening uptake}

During the study period (March to December 2018), 24230 adults ( $\geq 18$ years) were screened for HCV, of whom 1194 (5\%) were seropositive. Among the seropositive cases, 793 (66\%) were viraemic. While passive screening of the adult population ( $\geq 18$ years) was conducted of 9049 people between March and August 2018, combined active ( $\geq 45$ years) and passive ( $\geq 18$ years) screening covered 15181 individuals between October and December 2018. A higher proportion of seropositive cases in the $\geq 45$-year age group (12.4\%) was detected with passive screening than with combined active and passive screening (3.7\%); however, 14448 people were screened with combined passive and active screening and only 4396 with passive screening. During the entire study period and with each of the screening modalities, the NNS for detecting one $\mathrm{HCV}$ viraemic case in the age group $\geq 45$ years ( 11 for passive and 43 for active) was lower than that for the age group 18-44 years old (95 for passive and 56 for active) (Table 1). 
Fig. 1. Numbers of cases screened in the passive and active screening programmes by age group and month, Maung Russey operational district, Cambodia, March-December 2018

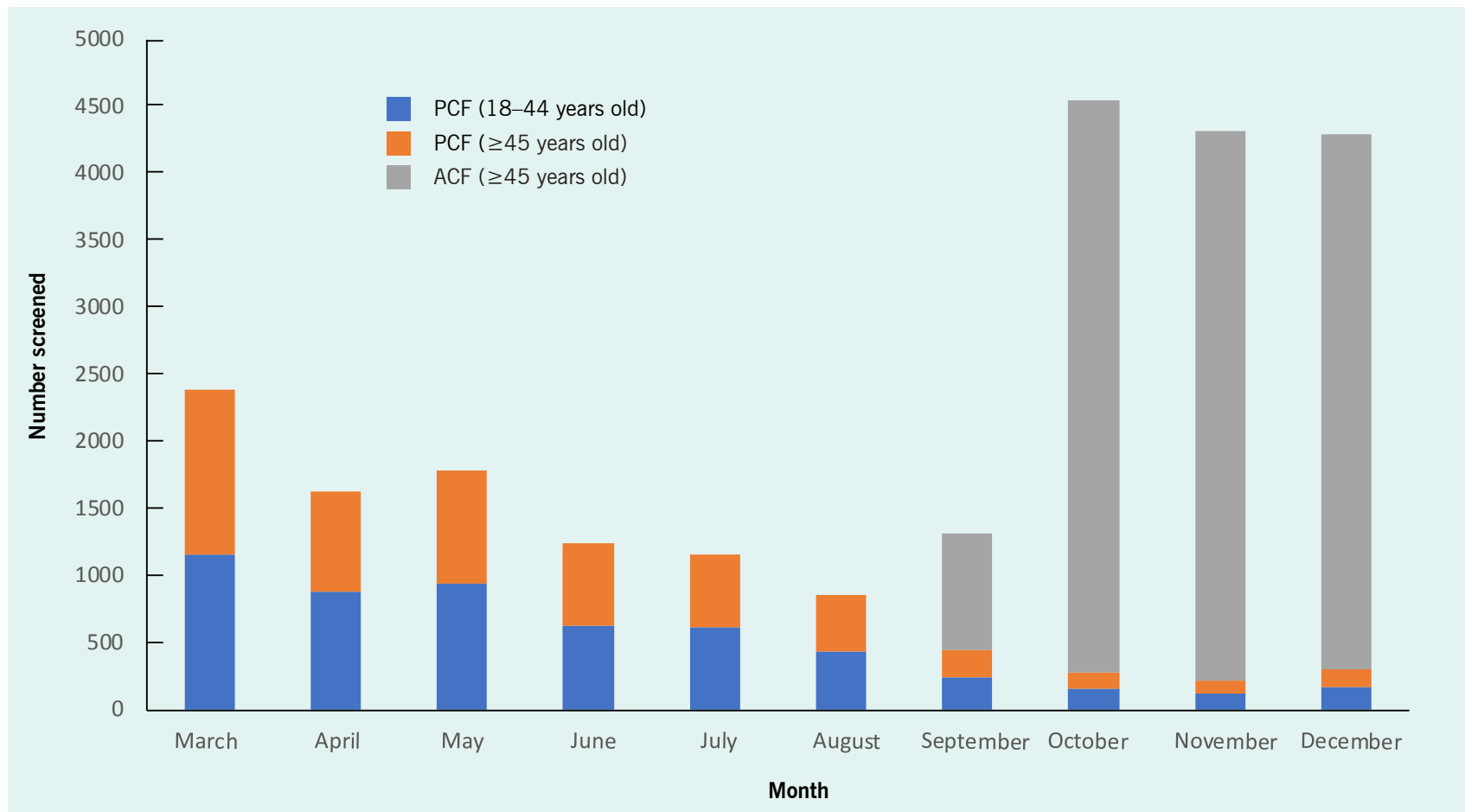

PCF: passive case finding; ACF: active case finding.

Table 1. Outcomes of passive and active screening in Maung Russey operational district, Cambodia, March-December 2018

\begin{tabular}{|c|c|c|c|c|c|c|}
\hline \multirow[b]{2}{*}{ Age group } & \multirow{2}{*}{$\begin{array}{c}\text { Total screened } \\
\text { N }\end{array}$} & \multicolumn{2}{|c|}{ Seropositive cases } & \multicolumn{3}{|c|}{ Viraemic cases } \\
\hline & & n & $\begin{array}{l}\text { (\% of total } \\
\text { screened) }\end{array}$ & n & $\begin{array}{c}\text { (\% of seropositive } \\
\text { cases) }\end{array}$ & NNS \\
\hline \multicolumn{7}{|c|}{ Phase 1: Passive screening (March-August) } \\
\hline 18-44 years & 4653 & 78 & $1.7 \%$ & 49 & $62.8 \%$ & 95 \\
\hline$\geq 45$ years & 4396 & 547 & $12.4 \%$ & 391 & $71.5 \%$ & 11 \\
\hline Total & 9049 & 625 & $6.9 \%$ & 440 & $70.4 \%$ & 21 \\
\hline \multicolumn{7}{|c|}{ Phase 2: Passive and active screening (September-December) } \\
\hline 18-44 years & 733 & 32 & $4.4 \%$ & 13 & $40.6 \%$ & 56 \\
\hline$\geq 45$ years & 14448 & 537 & $3.7 \%$ & 340 & $63.3 \%$ & 43 \\
\hline Total & 15181 & 569 & $3.7 \%$ & 353 & $62.0 \%$ & 43 \\
\hline \multicolumn{7}{|c|}{ Both phases (March-December) } \\
\hline Total & 24230 & 1194 & $4.9 \%$ & 793 & $66.4 \%$ & 31 \\
\hline
\end{tabular}

NNS: number needed to screen. 


\section{Screening effectiveness}

Fig. 1 shows the numbers of cases screened by age group and month in the passive and active screening programmes between March and December 2018. Combined passive and active screening covered $18 \%$ of the target population, while passive screening covered only $8 \%$ (Fig. 2). The proportions of seropositive and viraemic cases to the estimated number of cases were also higher for combined passive and active screening ( $45 \%$ and $41 \%$, respectively) than for passive screening alone (29\% and $28 \%$, respectively).

\section{Cost of the screening programme}

Between March and December, the programme cost US\$ 85208 for passive screening and US\$ 99733 for active and passive screening. The cost per viraemic positive case was US\$ 194 for passive screening and US\$ 283 for combined passive and active screening. The largest cost contributors were labour (31\%), tests and materials (29\%) and indirect costs (25\%) (Table 2).

\section{DISCUSSION}

The two HCV screening strategies tested in Maung Russey operational district in Cambodia detected 1194 (5\%) seropositive cases, $793(66 \%)$ of whom were viraemic. The programme cost a total of US $\$ 184941$, with a cost per case screened of US\$194 for passive screening and US\$ 283 for combined passive and active screening. To our knowledge, this is the first HCV screening intervention based in primary health care centres and at the community level in Cambodia. The findings provide reliable evidence for policy and actions to scale up HCV case-finding in Cambodia.

Screening for HCV was voluntary, and 24230 people were tested between March and December 2018 (18\% of 133162 targeted population). This relatively low screening rate might be due to low awareness of $\mathrm{HCV}$ in the community. A recent cross-sectional sero-survey reported that more than $64 \%$ of respondents were unaware of $\mathrm{HCV}^{6}{ }^{6}$ Moreover, the asymptomatic nature of $\mathrm{HCV}$ infection might mean that people do not seek testing. Our screening programme did not include health promotion; however, information on HCV screening and treatment was provided to all eligible patients during their health centre visit. The low coverage of active screening might be due to the fact that screening was conducted on only one day per village, so that people who were not present on that day missed the opportunity. Extending the number of community screening days, a strategy that has been used in other countries such as Rwanda, ${ }^{12}$ might increase participation. It is thus recommended that the national programme conduct active community screening for several days or organize annual catch-up screening campaigns.

We found that active screening greatly increased community screening coverage. While passive screening at health centres may reach symptomatic patients and those with known HCV status, active screening targets people outside health centres. Egypt, Rwanda and Taiwan (China) have implemented mass screening campaigns for the general population at community facilities such as stadiums and schools. ${ }^{11,12,14}$ However, the entire general population was screened during such mass campaigns, while in this study active case finding was conducted only among people aged $\geq 45$ years, who are known to be at high risk. ${ }^{6}$ In Cambodia, use of other locations for reaching older people and other high-risk groups might be more affordable and feasible. ${ }^{15,16}$ We also found that the NNS for finding one HCV case is lower when high-risk groups are targeted.

While the project was expected to achieve a higher seropositivity rate through active screening of adults aged $\geq 45$ years, the rate in the same age group was higher with passive screening. The probable explanation is that people visiting health centres are either aware of their HCV status or are symptomatic or at a late stage and seeking care. Again, the higher positivity rate among older people supports active screening of these groups in a later phase of the project.

We considered seven categories of costs, some of which can be removed from future costing by changing the way screening is conducted. For example, integrating HCV screening with TB screening at all levels (health centres, district, provincial and national) would greatly reduce costs, such as for installing GeneXpert, transport and overhead. The cost of combined passive screening and active screening (US\$283) was only US\$ 89 higher than that for passive screening alone (US\$ 194) but yielded a higher viraemic case detection rate $(41 \%$ versus $28 \%$ viraemic cases, respectively). Moreover, the cost per viraemic case detected was comparable to the estimated 
Fig. 2. Effectiveness of passive and active screening in Maung Russey operational district, Cambodia, MarchDecember 2018

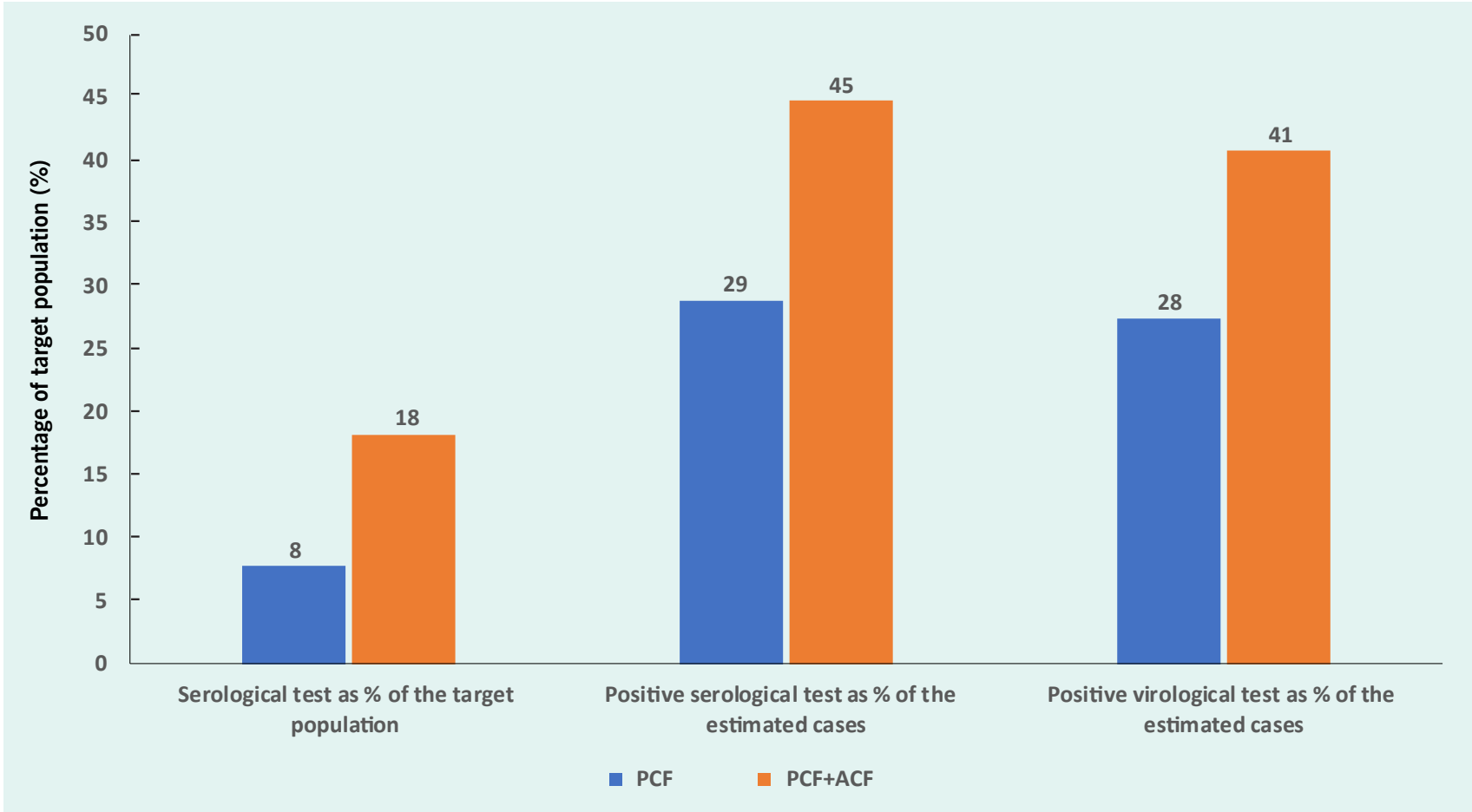

PCF: passive case finding; ACF: active case finding.

Table 2. Implementation costs of passive and active screening by cost category (US\$, 2018) in Maung Russey operational district, Cambodia, March-December 2018

\begin{tabular}{|c|c|c|c|c|c|}
\hline \multirow[b]{2}{*}{ Cost items } & \multicolumn{2}{|c|}{ Programme cost (US\$) } & \multicolumn{2}{|c|}{ Cost per case screened (US\$) } & \multirow[b]{2}{*}{ Cost profile (\%) } \\
\hline & $\begin{array}{c}\text { Passive } \\
\text { (Mar-Aug) }\end{array}$ & $\begin{array}{l}\text { Passive }+ \text { active } \\
\text { (Sep-Dec) }\end{array}$ & $\begin{array}{c}\text { Passive } \\
\text { (Mar-Aug) }\end{array}$ & $\begin{array}{l}\text { Passive }+ \text { active } \\
\text { (Sep-Dec) }\end{array}$ & \\
\hline Training ${ }^{1}$ & 1632 & 1632 & 3.7 & 4.6 & 2 \\
\hline Field equipment ${ }^{2}$ & 3352 & 3179 & 7.6 & 9.0 & 3 \\
\hline Labour $^{3}$ & 31791 & 31155 & 70.1 & 88.3 & 31 \\
\hline Transport ${ }^{4}$ & 18 & 11790 & 0.0 & 33.4 & 9 \\
\hline Tests and materials ${ }^{5}$ & 24906 & 28526 & 56.6 & 80.8 & 29 \\
\hline User fees ${ }^{6}$ & 762 & 1439 & 1.7 & 2.0 & 1 \\
\hline Indirect costs ${ }^{7}$ & 22747 & 22747 & 51.7 & 64.4 & 25 \\
\hline Total cost & 85208 & 99733 & 193.7 & 282.5 & 100 \\
\hline
\end{tabular}

${ }^{1}$ Cost of training both MSF and Ministry of Health health centre staff.

${ }^{2}$ GeneXpert machine, cold chain and other medical equipment used in the field.

${ }^{3}$ Labour costs include both MSF and government salaries.

${ }^{4}$ Renting of two cars and drivers for transport of active case finding team members and collected blood samples.

${ }^{5}$ Rapid diagnostic tests, viral load tests and other materials for blood collection and information, education and communication materials.

${ }^{6}$ MSF covered costs for uninsured patients to access tests other than HCV screening.

${ }^{7}$ Overhead costs (salaries, administration costs and other office operation costs). 
cost per TB case, which was US\$249 for active doorto-door screening in poor urban areas of Phnom Penh, US $\$ 308$ for testing of TB contacts and US\$316 for symptomatic testing of the older rural population. ${ }^{17}$ Integration of HCV screening with community programmes such as for TB, HIV and malaria could improve health workforce capacity and reduce the national cost of different screening programmes. ${ }^{14}$ Another possible costsaving strategy would be to take advantage of community gatherings during national holidays, such as the water festival and Pchum Ben, as well as World Hepatitis Day (28 July), to conduct periodic mass screening campaigns and HCV-related education.

\section{Strengths and limitations}

A major strength of this study is that the output and cost data were collected and analysed with input from a field implementation programme. It thus captured real-world screening outcomes and costs. Moreover, having one implementing partner simplified the cost estimates.

A limitation of our study is that it is based on field experience and was not initially designed as operational research; it therefore relied on pooled data collected during the screening period. Moreover, we did not use common measures of effectiveness, such as quality-adjusted or disability-adjusted life years. The comparability of these findings with those of other studies is thus limited.

\section{CONCLUSION}

The combined passive and active screening approach cost US\$ 89 more than passive screening alone yet provided a higher yield of viraemic cases $(41 \%$ versus $28 \%$, respectively). Thus, adding active screening to passive screening should be considered, as it might contribute to the objective of HCV elimination by 2030 , as recommended by WHO.

\section{Contributors}

JPD, MLP and IP conceived the study. SMH and IP managed the data and did the statistical analysis. IP checked for consistency of the analysis. SMH wrote the first draft of the article, which was edited for consistency by JPD, MLP, IP, CS and KA and critically revised for important intellectual content by CS and KA. All authors reviewed and approved the final version.

\section{Ethical considerations}

The study received ethical approval from the Cambodian National Ethics Committee for Health Research, with reference no. 023 NECHR dated 7 February 2019.

As the data presented were collected as part of routine programmatic work and were anonymized, the protocol of the study was approved by MSF as fulfilling the exemption criteria set by the MSF Ethics Review Board for retrospective analyses of routinely collected data.

\section{Funding}

The activity was funded by Médecins Sans Frontières France.

\section{Conflicts of interest}

We declare no competing interests.

\section{References}

1. Global hepatitis report 2017. Geneva: World Health Organization; 2017. Available from: https://apps.who.int/iris/handle/10665/255016, accessed 17 June 2021.

2. Lim AG, Trickey A, Vickerman P. Screening strategies for hepatitis C virus. Hepatol Commun. 2019;3(3):321-4. doi:10.1002/ hep4.1330 pmid:30859145

3. Blach S, Zeuzem S, Manns M, Altraif I, Duberg A-S, Muljono DH et al.; The Polaris Observatory HCV Collaborators. Global prevalence and genotype distribution of hepatitis $C$ virus infection in 2015: a modelling study. Lancet Gastroenterol Hepatol. 2017;2(3):161-76. doi:10.1016/S2468-1253(16)30181-9 pmid:28404132

4. A population-based survey of hepatitis $C$ prevalence and risk factors in three rural districts in Western Cambodia 2019. In: MSF report on Cambodia hepatitis C programme. Paris: Médecins Sans Frontières.

5. Sreng B, Kimcheng H, Sovann LY, Huot E. Epidemiology of viral hepatitis and liver diseases in Cambodia. Euroasian J Hepatogastroenterol. 2015;5(1):30-3. doi:10.5005/jp-journals-10018-1125 pmid:29201682

6. Lynch E, Falq G, Sun C, Bunchhoeung PDT, Huerga H, Loarec A et al. Hepatitis $C$ viraemic and seroprevalence and risk factors for positivity in northwest Cambodia: a household cross-sectional serosurvey. BMC Infect Dis. 2021;21(1):223. doi:10.1186/s12879021-05826-0 pmid:33637051

7. Nouhin J, Iwamoto M, Prak S, Dousset JP, Phon K, Heng S et al. Molecular epidemiology of hepatitis $\mathrm{C}$ virus in Cambodia during 2016-2017. Sci Rep. 2019;9(1):7314. doi:10.1038/s41598-019 43785-4 pmid:31086236 
8. Narin P, Hamajima N, Kouy S, Hirosawa T, Eav S. Characteristics of liver cancer at Khmer-Soviet Friendship Hospital in Phnom Penh, Cambodia. Asian Pacific J Cancer Prev. 2015;16(1):35-9. doi:10.7314/APJCP.2015.16.1.35 pmid:25640378

9. Chassagne F, Rojas Rojas T, Bertani S, Bourdy G, Eav S, Ruiz $E$ et al. A 13-year retrospective study on primary liver cancer in Cambodia: A strikingly high hepatitis $\mathrm{C}$ occurrence among hepatocellular carcinoma cases. Oncology. 2016;91(2):106-16. doi:10.1159/000446398 pmid:27250992

10. National Strategic Plan for Viral Hepatitis B and C (NSP 20202024). Pnomh Penh: Ministry of Health Cambodia; 2020. Available at: http://cdcmoh.gov.kh/prevention-control/viral-hepatitis, accessed 17 June 2021.

11. Schroeder SE, Pedrana A, Scott N, Wilson D, Kuschel C, Aufegger $L$ et al. Innovative strategies for the elimination of viral hepatitis at a national level: A country case series. Liver Int. 2019;39(10):181836. doi:10.1111/liv.14222 pmid:31433902

12. Umutesi J, Liu CY, Penkunas MJ, Makuza JD, Ntihabose CK, Umuraza $S$ et al. Screening a nation for hepatitis $C$ virus elimination: A cross-sectional study on prevalence of hepatitis $C$ and associated risk factors in the Rwandan general population. BM Open. 2019;9(7):e029743. doi:10.1136/bmjopen-2019-029743 pmid:31272986
13. Cambodia Inter-censal Population Survey 2013. Phnom Penh: National Institute of Statistics, Ministry of Planning; 2014. Available from: http://ghdx.healthdata.org/record/cambodia-intercensal-population-survey-2013, accessed 17 June 2021.

14. WHO guidelines on hepatitis $B$ and $C$ testing. Geneva: World Health Organization; 2017. Available from: https://apps.who.int/iris/handle/10665/254621, accessed 17 June 2021.

15. Yi S, Mun P, Chhoun P, Chann N, Tuot S, Mburu G. Prevalence of and risk factors for hepatitis $C$ virus antibody among people who inject drugs in Cambodia: A national biological and behavioral survey. Harm Reduct J. 2019;16(1):29. doi:10.1186/s12954-019-0299-1 pmid:31036011

16. De Weggheleire A, An S, De Baetselier I, Soeung P, Keath H, So V et al. A cross-sectional study of hepatitis $\mathrm{C}$ among people living with HIV in Cambodia: Prevalence, risk factors, and potential for targeted screening. PLoS One. 2017;12(8):e0183530. doi:10.1371/journal. pone. 0183530 pmid:28832660

17. James R, Khim K, Boudarene L, Yoong J, Phalla C, Saint S et al. Tuberculosis active case finding in Cambodia: a pragmatic, costeffectiveness comparison of three implementation models. BMC Infect Dis. 2017;17(1):580. doi:10.1186/s12879-017-2670-8 pmid:28830372 